\title{
Design and Realization of Multi-stage Radio Frequency Power Amplifiers Based on GaN HEMT
}

\author{
Yan An ${ }^{1,}$, Yankai Liu ${ }^{1, b}$ \\ ${ }^{1}$ College of Computer and Information Engineering, Tianjin Normal University, \\ Tianjin 300387, China \\ aemail: 1825187226@qq.com, bemail: yankailiu@qq.com
}

Keywords: Radio Frequency Power Amplifier; L Frequency Band; Broadband

\begin{abstract}
This article provides the design of multi-stage radio frequency power amplifiers which adopt transistors made of semiconductor materials of the third generation on the basis of taking into consideration criterions referring to efficiency, bandwidth and linearity. The first step is to conduct an experimental research on the circuit of the single-tube radio frequency power amplifier, and then, establish a three-stage pre-driving circuit; and constituting a final power amplifier assembly; finally, perform cascading on the pre-driving circuit and the final-stage power driving circuit to form the final radio frequency large-power amplifier. The simulation result indicates that the linearity of the multi-stage is good, so the effect is good.
\end{abstract}

\section{Background and Significance of the Study}

As the requirements for wireless communication systems are increased, the study on wireless communication is deeper.As an important module of a transmitter, the performance of the radio frequency power amplifier, such as working bandwidth, output power, additional efficiency and linearity, plays an important role in influencing the quality of the wireless communication system. The continuous increase and expansion of information content of communication systems demand larger working bandwidth, longer wireless communication distance and larger output power for power amplifiers. The miniaturization of mobile communication terminals demands the increase of additional efficiency for power amplifiers. The development of the modulation technique impels power amplifiers to demand higher linearity.

\section{Design Plan of Multi-Stage Radio Frequency Power Amplifier}

\section{Design structure}

It is difficult for single-tube radio frequency power amplifiers to realize $55 \mathrm{~dB}$ power gain. Therefore, four-stage radio frequency power amplifiers are combined in the cascading manner to form the final power amplifier assembly in order to meet final criterions. The power driving manner is adopted in the preceding three stages, and a parallel structure of single-tube radio frequency power amplifiers of four stages is adopted in the final stage in order to provide final power push. In this way, the final circuit output end can generate signals of $380 \mathrm{~W}$.

\section{Functions and design of each part}

1) The preceding amplifiers provide sufficient power for the final module, and their gain can amplify the small input signals to the extent required in final push in the linear manner; output ports are isolators, guaranteeing good isolation from final amplifiers; in the meantime, the preceding amplifiers should guarantee good in-band flatness and the consistency of amplitude-phase characteristics.

2) Two 3dB bridges (shunts) are used for dividing the output power of the third-stage amplifier into four paths, respectively providing equal-amplitude power push for four final amplifiers.

3) The final amplifiers directly provide final output power for power amplification. One GaN power tube with an appropriate match of input and output broadbands can offer over 100W power output in the full band. 
4) Two 3dB bridges (combined circuit) are used for combining output signals of four final amplifiers. To ensure combination frequency, good amplitude and phase equalization are required for combiners. In the meantime, four input ports should be well isolated in order to effectively avoid the ineffectiveness of the assembly due to the failure of single final amplifier.

\section{Final Rf Power Amplifier Assembly}

\section{Structure design}

According to the method of establishing a final power amplifier assembly in the design plan, the established single-stage RF power amplifiers referred to in the previous chapter are combined into a final power amplifier assembly (of which the total output power can reach $380 \mathrm{~W}$ ) by a $3 \mathrm{~dB}$ power divider/combiner.Because the input signal power of the final amplifier module should keep consistent with the previous input signal power $(35 \mathrm{dBm}$, withdraw $2 \mathrm{dBm}$ at $1 \mathrm{dBm}$ compression point), the input signal power of the final power amplifier assembly must be approximately four times of the input signal power of single path, namely increasing 5-6dB, about $40 \mathrm{dBm}$.

Test of final power amplifier assembly on power in full range and efficiency

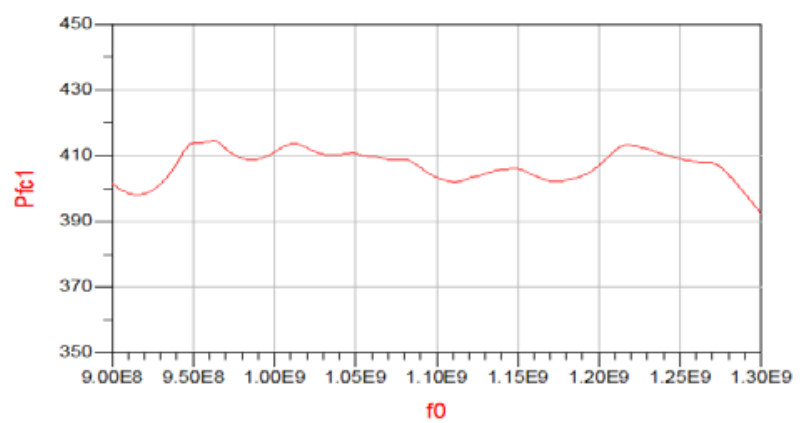

Fig.3.2-1. Input power is $40 \mathrm{dBm}$, output power changes with frequency

The figure 3.2-1 shows the changes of output power in the full range with frequency when the input power is $40 \mathrm{dBm}$. It indicates that the output power is $380 \mathrm{~W}$ in the full range (950MHz 1250MHz), meeting the criterion.

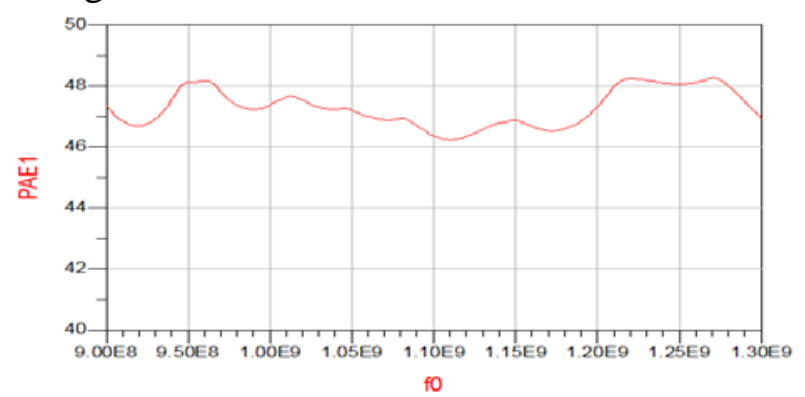

Fig.3.2-2. Input power is 40dBm, additional efficiency changes with frequency

The figure 3.2-2 shows the changes of additional efficiency (PAE) with frequency when the input power is $40 \mathrm{dBm}$. The changing curve indicates that the additional efficiency in the full range fluctuates between $46 \% \sim 48 \%$, which is larger than the criterion (38\%).

\section{Driving Amplifier Assembly at Preceding Stage}

\section{First-stage power amplifier}

AH125 is a transistor made of InGaP/GaAs semiconductor materials. Due to its internal bias, the transistor maintains high linearity during output, and only $+5 \mathrm{~V}$ single power supply is needed.

Figure 4.1-1.shows the first-stage amplifying circuit 


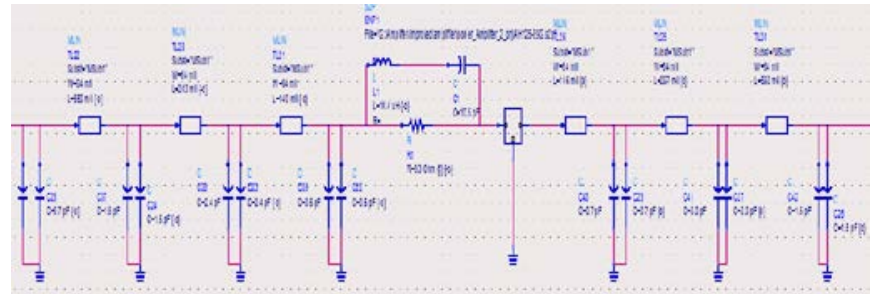

Fig.4.1-1. Whole circuit of first-stage amplifier

\section{Second-stage power amplifier}

The second-stage RF amplifier is designed by the CGH40006F GaN transistor produced by Cree. Figure 4.2-1 shows the second-stage RF amplifier circuit

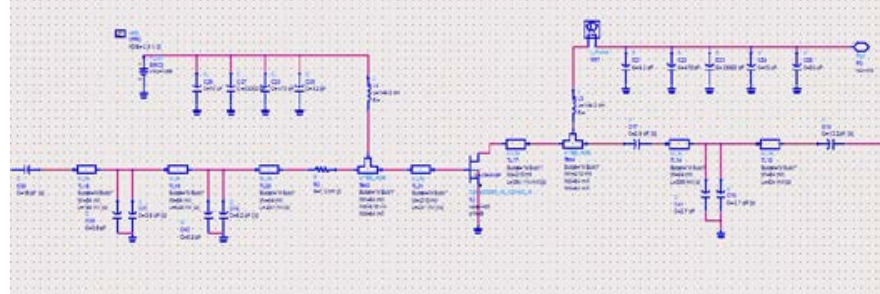

Fig.4.2-1. Whole circuit of second-stage amplifier

\section{Third-stage power smplifer}

The third-stage amplifying circuit adopts CGH40045 transistor of which the series and model are the same as those of the transistor adopted in the second-stage amplifier.

The figure 4.3-1 shows the whole circuit of the third-stage amplifier.

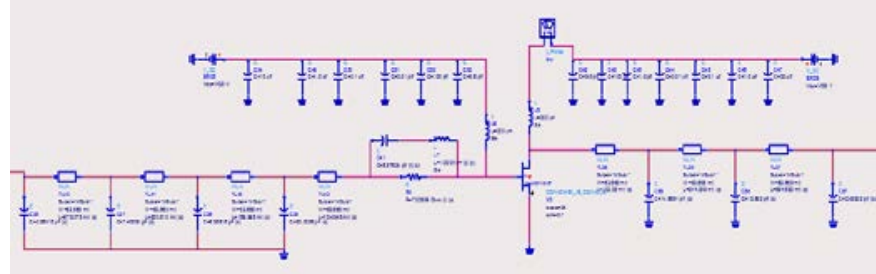

Fig.4.3-1. Whole circuit of third-stage amplifier

\section{Whole circuit test}

Figure 4.4-1 shows the circuit of the whole power amplifier formed by four cascaded modules.

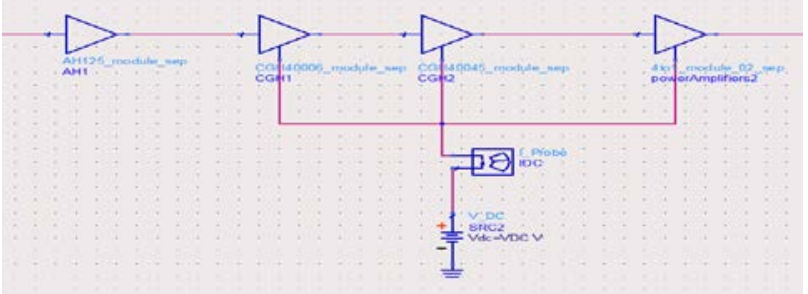

Fig.4.4-1. Circuit of multi-stage RF large-power amplifier

Two indexes of the power amplifier should be tested: the small signal gain and the voltage standing wave ratio. The figure 4.4-2 shows the $S(2,1)$ curve of the whole power amplifier, namely the curve of small signal gain. It indicates that the small signal gain in the whole working range $(950 \mathrm{MHz} 1250 \mathrm{MHz})$ is larger than $55 \mathrm{~dB}$, which meets the requirement. The figure $4.4-3$ shows the input voltage standing wave ratio of the power amplifier, which is mostly smaller than 2:1. Therefore, it meets the requirement. 


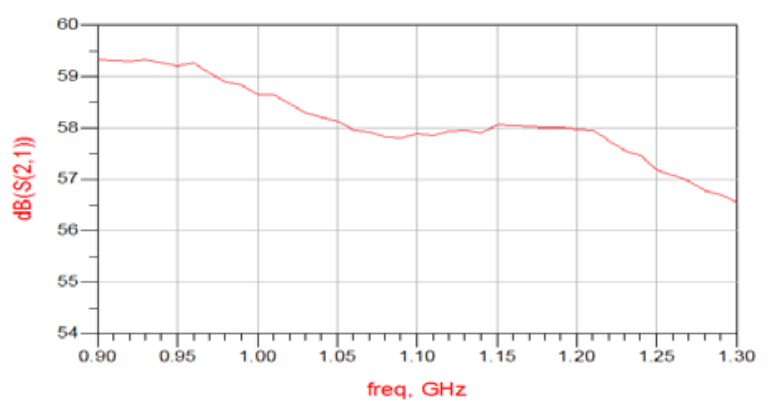

Fig.4.4-2. Small signal gain of whole circuit

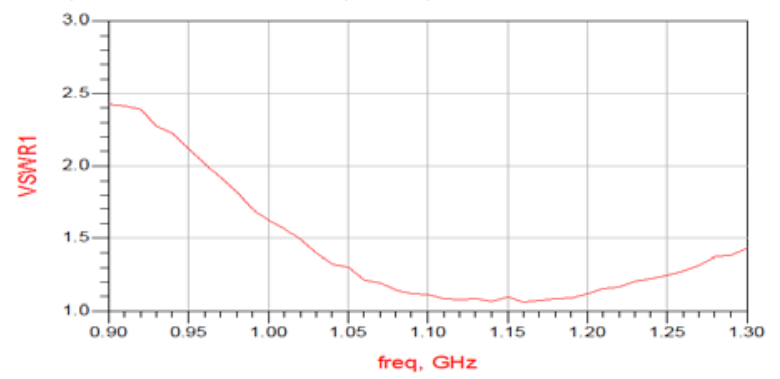

Fig.4.4-3. Input voltage standing wave ratio of whole circuit

\section{Summary and Outlook}

This article designs a multi-stage RF large-power amplifier, which is formed by cascading of three stages of amplifying circuits in the front and a final power amplifier. The amplifying circuits of the front thee stages respectively adopt AH125, CGH40006 and CGH40045 power tubes. The final power amplifier assembly is formed by the parallel connection of four single-tube power amplifier modules. Finally, the front amplifying circuits of three stages and the final power amplifier assembly are cascaded to form an original broadband large power amplifier. Then, the formed power amplifier is tested, and results meet the criterion. However, it is difficult to keep linearity same in the full range, so if the requirement for linearity is high, an additional circuit is still needed to improve the linearity.

\section{Acknowledgement}

Tianjin normal university college students' innovative entrepreneurial training project funding (project number: 201710065069, the level of project: the municipal).

\section{References}

[1] Leng Yongqing, Study of Characteristics of GaN Transistors with High Electron Mobility and Power Amplifier: [Doctor Dissertation], Hunan University, Changsha, 2013

[2] Arnous, Mhd.Tareq, Saad, Paul ,Preis, Sebastian , Zihui Zhang. Highly efficient and wideband harmonically tuned GaN-HEMT power amplifier. In: Microwaves, Radar, and Wireless Communication (MIKON), 2014 20th International Conference on. IEEE, 2014.1 4

[3] Chi C, Jun C, Lei W. L-Band High efficiency GaN HEMT Power Amplifier for Space Application, 2013: [Master Dissertation], Harbin Institute of Technology, Harbin, 2013

[4] Margomenos A, Kurdoghlian A, Micovic M, et al. 70-105 GHz wideband GaN power amplifiers. In:Microwave Integrated Circuits Conference (EuMIC), 2012 7th European. IEEE, 2012. 199 202

[5] Ding Yao, Study of High-Efficiency RF Power Amplifier: [Doctor Dissertation], (University of Science and Technology of China, Hefei, 2012 
[6] Gustafsson D, Andersson C M, Hellberg R, et a, A 44 dBm 1.0-3.0 GHz GaN power amplifier with over 45\% PAE at $6 \mathrm{~dB}$ back-off, Microwave Symposium Digest (IMS), 2013 IEEE MTT-S International. IEEE, 2013. 1 4

[7] Arnous M T, Bathich K, Preis S, et al. $100 \mathrm{~W}$ highly efficient octave bandwidth GaN-HEMT power amplifier, Microwave Radar and Wireless Communications (MIKON), 2012 19th International Conference on, IEEE, 2012, 1: 289 292

[8] Sang Lei, Modeling Research of AlGaN/GaN HEMT Power Devices and Design of High Efficiency Amplifier: [Doctor Dissertation], University of Electronic Science and Technology of China, Chengdu, 2013

[9] Sun Shitao, Study of GaN High-efficiency Power Amplifier: [Master Dissertation], Hefei University of Technology, Hefei, 2014

[10] Hayat K, Kashif A, Azam S, et al. High performance GaN HEMT class-AB RF power amplifier for L-band application. In: Applied Sciences and Technology (IBCAST), 2013 10th International Bhurban Conference on. IEEE, 2013. 389 392

[11] Fu Wenli, Study of New Structure and Model of GaN HEMT Power Device: [Master Dissertation], University of Electronic Science and Technology of China, Chengdu, 2013

[12] Gustafsson D, Andersson C M, Hellberg R, et al. A 44 dBm 1.0-3.0 GHz GaN power amplifier with over 45\% PAE at 6 dB back-off. In: Microwave Symposium Digest (IMS), 2013 IEEE MTT-S International. IEEE, 2013: 1 4

[13] Saad P, Nemati H M, Andersson K, et al. Highly efficient GaN-HEMT power amplifiers at 3.5 GHz and 5.5 GHz. In: Wireless and Microwave Technology Conference (WAMICON), 2011 IEEE 12th Annual. IEEE, 2011. 1 4 